Research Article

\title{
Remaining Useful Life Prediction of Rolling Bearings Using Electrostatic Monitoring Based on Two-Stage Information Fusion Stochastic Filtering
}

\author{
Ying Zhang $\mathbb{D}^{1,2}$ and Anchen Wang ${ }^{1}$ \\ ${ }^{1}$ College of Automobile and Traffific Engineering, Nanjing Forestry University, Nanjing 210037, China \\ ${ }^{2}$ College of Civil Aviation, Nanjing University of Aeronautics and Astronautics, Nanjing 210037, China \\ Correspondence should be addressed to Ying Zhang; zhangyingrms@163.com
}

Received 25 October 2019; Revised 13 January 2020; Accepted 8 February 2020; Published 17 March 2020

Academic Editor: Jose de Jesus Rubio

Copyright (c) 2020 Ying Zhang and Anchen Wang. This is an open access article distributed under the Creative Commons Attribution License, which permits unrestricted use, distribution, and reproduction in any medium, provided the original work is properly cited.

\begin{abstract}
The accurate prediction of the remaining useful life (RUL) of rolling bearings is of great significance for a rational formulation of maintenance strategies and the reduction of maintenance costs. According to the two-stage nonlinear degradation characteristics of rolling bearing operation, this paper proposes a prognosis model based on modified stochastic filtering. First, multiple features reextracted from the time domain, frequency domain, and complexity angles, and the baseline Gaussian mixture model (GMM) is established using the normal operating data after spectral regression. The Bayesian-inferred distance (BID) is used as a quantitative indicator to reflect the bearing performance degradation degree. Then, taking multiparameter fusion results as input, the relationship between BID and remaining life is established by the two-stage stochastic filtering model to realize online dynamic remaining useful life prediction. The method in this paper overcomes the difficulty of accurately defining the failure threshold of rolling bearing. At the same time, it reduces the computational burden, avoiding the need of calculating the joint probability distribution for high-dimensional data. Finally, the proposed method has been verified experimentally to have high precision and engineering application value.
\end{abstract}

\section{Introduction}

As key components of rotating equipment, the performance degradation and failure of rolling bearings affect the performance of the machine and even may lead to unplanned equipment shutdown. However, due to the uncertainty of the working environment and conditions of rolling bearings, the randomness of the development of fatigue damage and the diversity of failure modes lead to the large discrete life of rolling bearing, and regular maintenance often results in "undermaintenance" or "overmaintenance". In order to ensure the safety, prognostic and health management (PHM) of the rolling bearing can maximize the bearing useful life and reduce the maintenance cost. The accurate acquisition of bearing status information is a prerequisite to realize the PHM of rolling bearings and a reliable and effective method for predicting the remaining useful life is an important means.

In terms of state information acquisition, the electrostatic monitoring technology provides a high-sensitivity online monitoring method for rolling bearings. Physical and chemical changes accompany the rolling bearing wear process. At the same time, it generates a large number of abrasive particles that cause electrostatic induction. Monitoring the change in the total amount of electrostatic charge in the wear zone can accurately reflect the state of bearing degradation $[1,2]$. Unlike vibration and temperature, which are secondary effects of degradation, electrostatic monitoring is direct monitoring of contact degradation to provide support for accurate acquisition of bearing status information [3]. In the research of electrostatic induction mechanism, Tasbaz et al. [4] used a region wear electrostatic 
sensor and a friction coefficient to monitor and study point contact sliding friction gluing failure on a pin-on-disc and a reciprocating laboratory wear rig. The research results showed that the electrostatic sensor detects abnormality before the fault, which is better than the friction coefficient and more suitable for the online monitoring of wear faults. Morris et al. [5] studied the sliding friction electrostatic monitoring experiment under nonlubricated conditions. The research results showed that the electrostatic signal amplitude is consistent with the change in the amount of wear and the change in the friction coefficient. Wang et al. [6] conducted an online monitoring experiment on the point contact sliding friction of silicon nitride and various bearing steels for the friction and wear problems of composite materials that may be used in the new type of bearings and compared and analyzed the relationship between the wear amount, charge generation, friction coefficient, contact temperature, and surface roughness. Wang et al. [7] performed sliding friction experiments of point contact under different conditions for the wear of ceramics and bearing steels. The research results showed that there is a relationship between the volume loss of ceramic balls and the RMS value of electrostatic signals in the normal wear stage.

According to the current literature, the RUL prediction can be mainly divided into three categories: model-based life prediction technology, artificial intelligence, and statisticalbased life prediction [8]. The model-based life prediction technology relies on the degradation mechanism of the system. In order to predict the RUL of rolling bearings, by using the Paris formula, Qian et al. [9] established a physical prediction model for describing the crack propagation process. Kacprzynski et al. [10] extended the fracture mechanics model and the crack initiation model based on the data fusion algorithm and parameter adjustment and verified the prediction experimentally. Liu and Mahadevan [11] applied the fatigue life prediction model to the crack propagation stage for predicting the fatigue life in the crack initiation stage by the equivalent initial crack size method. Also, they established a remaining life prediction model for the uniform crack initiation and expansion stage. Artificial intelligence-based life prediction methods use machine learning methods to fit the degradation process of the system and then predict the RUL. Neural network and its many derivative algorithms $[12,13]$ are important technologies in the field of artificial intelligence. They can be applied not only to classification problems [14], but also to prediction of remaining useful life. Yang et al. [15] proposed a prediction model based on the double-layer convolutional neural network $(\mathrm{CNN})$ to predict the remaining useful life of the bearing. Carrol et al. [16] used ANN, support vector machine (SVM), and logistic regression to predict the remaining life of a gearbox and found that the ANN method has the highest prediction accuracy. Li et al. [17] established a RUL prediction model for lithium batteries based on Elman neural network and verified the feasibility of the model in prediction. Ordonez et al. [18] proposed a prediction model based on kernel principal component analysis (KPCA) and gated recursive unit (GRU) suitable for the life prediction of complex systems and verified the accuracy of the method through aeronautical propulsion system simulation data. Wang et al. [19] proposed a two-dimensional $\mathrm{CNN}$ for predicting the RUL of rolling bearings and proved the high accuracy and fast calculation speed of the method by experiments. The statistical-based life prediction method models the data with a stochastic model to achieve RUL prediction. Mishra and Vanli [20] proposed a prediction model based on the principal component regression and Wiener process degradation model to predict the remaining life of a sensor. In order to avoid the need for subjective selection of degradation indicator and first prediction time in the Wiener process, Aisong et al. [21] proposed a Wiener process model for earthquake prediction based on optimal degradation indicator. Particle filtering [22-24], stochastic filtering [25], Kalman filter [26], and other filtering algorithms can also be used to predict the remaining useful life. Wang et al. [22] established a spherical cubic particle filter (SCPF) model to predict the remaining life of lithium-ion batteries and verified the effectiveness of the method. Lei et al. [23] used the maximum likelihood estimation algorithm to initialize the model parameters and proposed a particle filter-based algorithm to predict the remaining life of rolling bearings. Catterson et al. [24] predicted the remaining life of a transformer through a particle filter model.

However, for rolling bearings, due to the difficulty in obtaining an accurate failure threshold and the absence of a standard to follow, the final failure state can be observed. That is, life data is available. Life prediction methods that require the identification of a failure threshold, such as time series prediction, gray prediction theory, and particle filtering, are difficult to apply. The hidden Markov model does not need a failure threshold, but the training of the model requires artificially dividing the life cycle of the rolling bearing into several stages, which is subjective and difficult to grasp. The stochastic filtering model does not need a default failure threshold [25], and it can synthesize the current and historical state monitoring information to predict the RUL and calculate the probability density distribution of the RUL. This model is then more suitable for decision making of maintenance, and it requires singledimensional input data. If the input data is multidimensional, the calculation amount of joint probability density distribution is large, and it is difficult to realize the online real-time residual prediction. However, a single parameter such as RMS may not describe easily the state of the rolling bearing with accuracy. In our previous research [27], a multiparameter fusion performance evaluation model based on the spectral regression-Gaussian mixture model (SRGMM) was proposed to overcome the insensitivity or inconsistency of the single characteristic parameter to the bearing performance degradation. The proposed method integrates multiple characteristic parameters of electrostatic monitoring and can detect the occurrence of bearing degradation earlier.

The life of bearings includes two stages: normal operating stage and defective operating stage. In the second stage, equipment defect is launched, with the continuous work of equipment into a fault. It is important to obtain the starting 
point of the defective operating stage and predict its RUL. Wang [25] divided the operation of the equipment into two stages by using the concept of delay time. The transition between states was constructed by using the hidden Markov model, and the relationship between state information and device state was constructed by using the stochastic filtering theory. Wang et al. [28] used $\mu+3 \sigma$ to segment the two stages of the bearing degradation process, taking 14 timedomain characteristic parameters as inputs and using the prediction stage based on Kalman filtering to estimate the remaining useful life of the bearing. Wang and Tsui [29] established a statistical two-stage model of bearing degradation signal based on the seven harmonics of a bearing fault characteristic frequency. The statistical model of normal working stage is used to obtain the point at which the bearing begins to degenerate, and the statistical model of failure delay stage is used to predict the RUL of the bearing.

Based on single parameter random filter life prediction model [25], this paper proposes a remaining useful life prediction method of rolling bearings using electrostatic monitoring based on two-stage information fusion stochastic filtering. This method establishes a multiparameter fused health factor as a quantitative index reflecting bearing performance degradation. Based on this, the time of defect occurrence is obtained using the kernel density estimation (KDE) method, that is, the time point of distinguishing between two stages. Subsequently, based on stochastic filtering theory, the quantitative index of multiparameter fusion is taken as an input, and the relationship between the bearing monitoring index and the remaining life with twostage characteristic is established, so as to realize the online dynamic remaining life prediction. The method overcomes the difficulty of identifying a failure threshold, the absence of a standard to follow, the need of single-dimensional input data, and the high calculation cost of multidimensional input data. Finally, the validity and accuracy of the proposed method are verified by experiments.

\section{Description of Rolling Bearing Useful Life Prediction}

\subsection{Stages and Characteristics of Rolling Bearing Operation.}

The operating process of the bearing has two stages. The rolling bearing operation can be generally divided into normal operating condition and defective operating condition. The defective operating condition spans from the beginning of the early degradation to the severe degradation until the final complete failure. Figure 1 shows the result of a multiparameter fusion related to a lifetime test bearing [27]. The monitoring indicator of the bearing in normal operating condition is relatively stable and fluctuates randomly within a certain range. When entering the defective operating stage, the monitoring indicator changes (rises or decreases), and abnormal fluctuation accompanies the transition. The variation increases as the deterioration of bearing performance increases. This phenomenon has similar characteristics with conventional indicators such as RMS and kurtosis.

The measured signal is random. Due to the complexity of the working environment, bearing failure mechanism, and the interference of noise, the monitoring indicators are also random. Therefore, especially in the defect operating stage, the value of the current monitoring indicator cannot simply reflect the degradation degree of bearing performance, and historical monitoring data should be considered. In Figure 1, it can be observed that the monitoring indicator has a strong randomness. In the first stage, the fluctuation is stable. In the second stage, it rises while fluctuating. The real-time monitoring signal of the bearing contains not only healthstage information but also measurement noise [30]. The existence of these noises (deviations) is unavoidable, and some methods can be adopted to reduce the impact of the deviations on the monitoring results, such as using particle filtering $(\mathrm{PF})$ to reduce the random errors in the stochastic process [31].

The operating state of the bearing is hidden. During the operation of bearings, only the fault state of the bearing can be directly observed, while the normal operating state and defect operating state of the bearing cannot be directly observed. Though the degradation state of the bearing performance is unobservable, it can be inferred from the monitoring indicator. That is, there is observability of the monitoring data and an unobservability of the actual state.

2.2. Remaining Useful Life Prediction. In industrial production, the operator needs to know both the current degradation degree of the equipment and the equipmentestimated RUL to arrange a repair. The two stages of the rolling bearings operating state result in an inconsistent relationship between monitoring indicators and RUL in different operating states. In normal operating conditions, the monitoring indicator fluctuates randomly within a certain range, and the RUL decreases with the increase of running time. It is not easy to determine the relationship between the monitoring indicator and RUL. In the defect operating stage, although there are some fluctuations, the monitoring indicator has obvious trends. For example, the multiparameter fusion indicator BID has an upward trend in the defect operating stage while the RUL decreases with the increase of running time. By establishing the relationship between monitoring indicator and remaining life, the RUL can be obtained through the current time and historical monitoring data. Therefore, to predict the RUL, the occurrence time point of the defect $(u)$ and the relationship between monitoring data and lifetime are necessary. There is serious nonlinearity in the degradation process of bearings. The traditional parameter estimation method cannot be used to estimate it. However, KDE does not need to know the prior knowledge of data distribution and does not attach any assumptions to the data distribution. It is a nonparametric estimation method to study the characteristics of data distribution from the data sample itself, so this paper uses KDE to calculate the control limit. When the bearing is in the normal state, the BID value of the health factor fluctuates steadily. In this paper, it is set that the bearing begins to degrade when it exceeds the confidence interval of $99 \%$. This research determines the time point $u$ by the KDE method [27] and trains the degradation stage model through the 


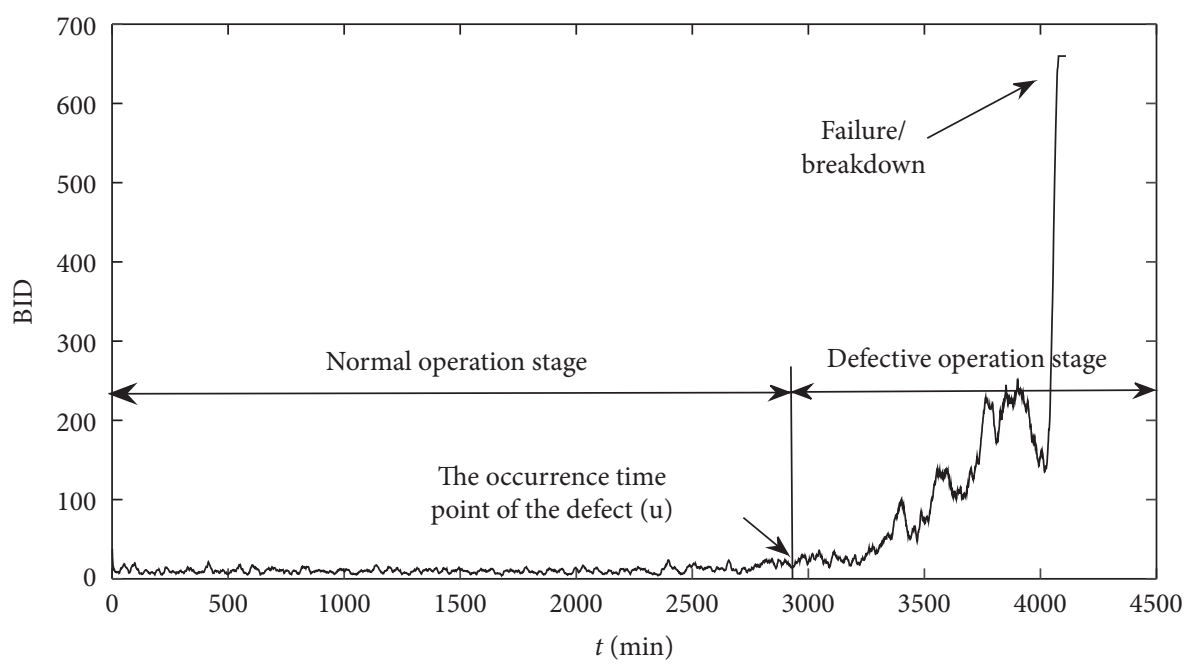

FIgUre 1: Estimate value of BID for bearing 1.

defect occurrence and development stage data to predict the remaining life of the defect stage.

From the monitoring indicators, especially in the defect operating stage, the data have some random fluctuations instead of a monotonous trend. Therefore, current data alone will cause misjudgment of the bearing performance degradation state and affect the accuracy of remaining life prediction. A given failure threshold is not essential in the stochastic filtering model. This model predicts the remaining life by combining current and historical state monitoring information and calculates the distribution of probability density of remaining life. The results suggest that the model is more suitable for maintenance decision making. However, the stochastic filtering model requires single-dimensional input data. If the input data is multidimensional, the calculation amount of joint probability density distribution is large, and it is difficult to realize the online real-time RUL prediction. Therefore, this paper proposes a two-stage RUL prediction framework based on multiparameter fusion, that is, the multiparameter fusion result BID is set as the input to establish the relationship between the defect stage monitoring indicator and the remaining life, thereby realizing the online dynamic RUL prediction.

\section{Theoretical Basis}

3.1. Construction of Health Indicators. Bearings play a key role in rotating machinery, and its health status is worthy of attention in practice. However, it is difficult to quantitatively analyze the health status of bearing in many cases, and the construction of health indicators can be used to evaluate the current health [32]. In our previous work [27], conventional time and frequency domain indicator for electrostatic monitoring cannot sufficiently reflect the degradation of rolling bearing performance. Since the random component in the electrostatic signal changes continuously as the performance of the rolling bearing deteriorates, the complexity measurement method is introduced to describe this process. To overcome the insensitivity and inconsistency of a single characteristic parameter with the bearing performance degradation, we introduced a multiparameter fusion performance evaluation model based on SR-GMM.

Firstly, 15 conventional time domain and frequency domain indicators and 3 complexity metrics indicators (approximate entropy, sample entropy, and arrangement entropy) were extracted as characteristic parameters to describe the degradation process of bearing from normal state to fault state. Time and frequency domain indexes are as follows:

\subsubsection{Time-Domain Indicators}

$$
\begin{aligned}
\text { Root mean square: } x_{\mathrm{rms}} & =\sqrt{\frac{\sum_{n=1}^{N}(x(n))^{2}}{N}}, \\
\text { Standard deviation: } x_{\mathrm{std}} & =\sqrt{\frac{\sum_{n=1}^{N}\left(x(n)-x_{m}\right)^{2}}{N-1},} \\
\text { Peak-Peak: } x_{p-p} & =\max \left(x_{n}\right)-\min \left(x_{n}\right), \\
\text { Skewness: } x_{\mathrm{ske}} & =\frac{\sum_{n=1}^{N}\left(x(n)-x_{m}\right)^{3}}{(N-1) x_{\mathrm{std}}^{3}}, \\
\text { Kurtosis: } x_{\mathrm{kur}} & =\frac{\sum_{n=1}^{N}\left(x(n)-x_{m}\right)^{4}}{(N-1) x_{\mathrm{std}}^{4}}, \\
\text { Crest factor: } \mathrm{CF} & =\frac{x_{p}}{x_{\mathrm{rms}}}, \\
\text { Shape factor: } \mathrm{SF} & =\frac{x_{p}}{\left(1 / N \sum_{n=1}^{N}|x(n)|\right)^{2}}, \\
\text { Clearance factor: } \mathrm{CLF} & =\frac{x_{\mathrm{rms}}}{\left(1 / N \sum_{n=1}^{N} \sqrt{|x(n)|}\right)^{2}}, \\
& \frac{x_{p}}{\left(1 / N \sum_{n=1}^{N}|x(n)|\right)},
\end{aligned}
$$

where $x(n)$ represents the amplitude of the signal. 


\subsubsection{Frequency Domain Indicators}

$$
\text { Frequency center: } x_{\mathrm{fc}}=\frac{\sum_{k=1}^{K} f_{k} s(k)}{\sum_{k=1}^{K} s(k)}
$$

Root mean square frequency: $x_{\mathrm{rmsf}}=\sqrt{\frac{\sum_{k=1}^{K} f_{k}^{2} s(k)}{\sum_{k=1}^{K} s(k)}}$,

Standard deviation frequency: $x_{\mathrm{stdf}}=\sqrt{\frac{\sum_{k=1}^{K}\left(f_{k}-x\right)_{\mathrm{fc}}^{2} s(k)}{\sum_{k=1}^{K} s(k)}}$,

Spectrum peak ratio inner: SPRI $=\frac{\sum_{i=1}^{H} p_{I}(h) k}{\sum_{k=1}^{K} s(k)}$,

Spectrum peak ratio outer: SPRO $=\frac{k \sum_{i=1}^{H} p_{O}(h)}{\sum_{i=1}^{H} s(k)}$,

Spectrum peak ratio of ball: SPRB $=\frac{k \sum_{i=1}^{H} p_{B}(h)}{\sum_{k=1}^{K} s(k)}$,

$s(k)$ represents the amplitude of the signal, $k=1,2, \ldots, K, K$ is the number of spectral lines, and $f_{k}$ is the frequency of spectral line $k$.

3.1.3. Health Index Construction. Then, spectral regression is used to reduce the dimension of the characteristic parameter data. The baseline GMM model is established with the normal operating condition data after spectral regression, and the BID index between the test data and the baseline GMM model is used as a quantitative indicator reflecting the degree of degradation of bearing performance.

The health indicator construction of multiparameter fusion consists of offline modeling and online evaluation. The details of the process are as follows (Figure 2):

\section{(1) Offline}

(1) Signal Denoising. The denoising process of normal operating condition data is performed by the joint denoising method based on spectral interpolation and singular value difference spectrum.

(2) The characteristic parameters are extracted, and the dimension of the original characteristic space is reduced by SR.

(3) Select the normal state data to establish a baseline GMM model and obtain the parameters of the model.

(4) Calculate BID. The moving average method is used to smooth the indicator to improve the sensitivity and reliability of the indicator:

$$
y(i)=\frac{1}{M} \sum_{j=0}^{M-1} x(i+j)
$$

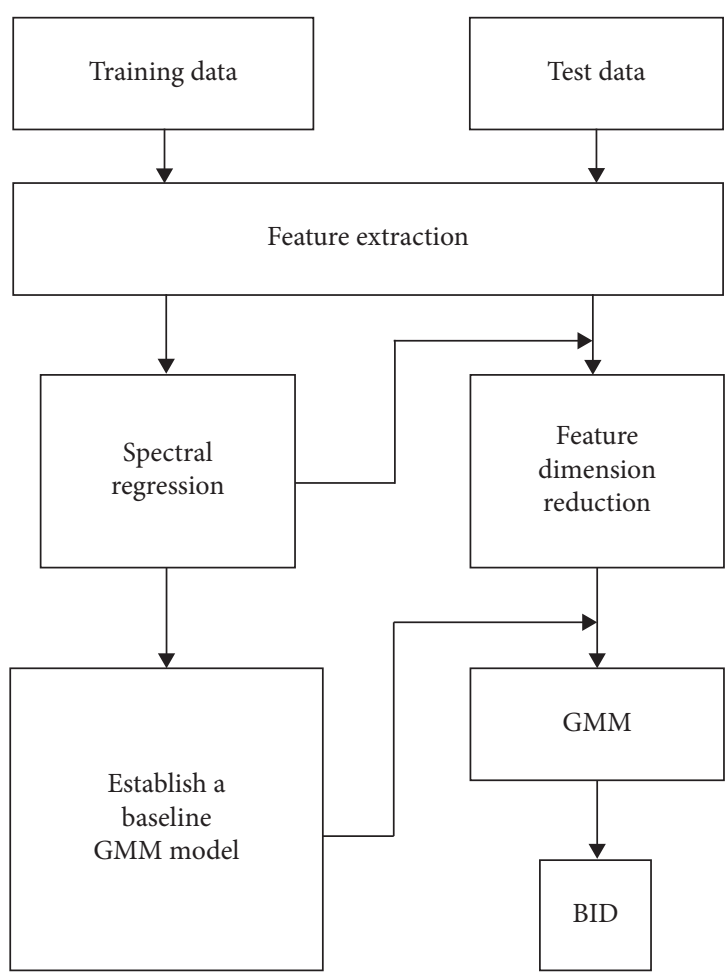

FIGURE 2: Multiparameter fusion process.

where $x(i)$ is input signal, $y(i)$ is the output signal, and $M$ is the moving average point set to 5 in this paper.

(5) Establish the control line. KDE is used to establish the control limits of the indicator to trigger the alarm at a slight degradation:

$$
\widehat{f}(x, H)=\frac{1}{n} \sum_{i=1}^{n} K\left(H^{-1 / 2}\left(x-x_{i}\right)\right) .
$$

(2) Online

(1) The data to be tested are first denoised. Then, the characteristic parameters are extracted (Step 1 in offline modeling), and the dimension is reduced (Step 2).

(2) The distance BID between the test data and the normal operating condition baseline the GMM model is calculated as a quantitative health indicator for the rolling bearing state.

(3) By comparing the relationship between the BID and the control line, the time when the bearing enters the degrading state is determined, and the bearing performance state can be quantitatively evaluated.

\subsection{RUL Prediction Method Based on Stochastic Filtering}

3.2.1. Stochastic Filtering. Stochastic filtering has the advantage of solving the randomness of measured information. 
Consider a stochastic dynamic system, which can be described by a state space model:

$$
\begin{aligned}
x_{t+1} & =\varphi\left(x_{t}, t\right)+\Gamma\left(x_{t}, t\right) w_{t+1}, \\
y_{t} & =h\left(x_{t}, t\right)+v_{t} .
\end{aligned}
$$

Equation (5) is the state equation, where $x_{t}$ is the $n$ dimensional state vector of the system at time $t, \varphi\left(x_{t}, t\right)$ is the $n$-dimensional vector function, $\Gamma\left(x_{t}, t\right)$ is the $n \times r$-dimensional vector, and $w_{t+1}$ is the $r$-dimensional stochastic state noise vector, which satisfies $w_{t+1} \sim N(0, Q(t+1))$, and $\mathrm{Q}(t+1)$ is the covariance matrix of $w_{t+1}$, that is, $w_{t+1}$ is a Gaussian white noise sequence. Under normal circumstances, the state of the system cannot be directly observed.

Equation (6) is the observation equation, where $y_{t}$ is the $m$-dimensional observation vector of the system at time $t$, $h\left(x_{t}, t\right)$ is the $m$-dimensional vector function, $v_{t}$ is the $m$ dimensional stochastic observation noise vector, which satisfies $v_{t} \sim N(0, R(t))$, and $R(t)$ is the covariance matrix of $v_{t}$, that is, $v_{t}$ is a Gaussian white noise sequence.

Suppose the distribution of the initial state $x_{0}$ of the monitored system is known, and $x_{0}, w_{t}$, and $v_{t}$ are independent of each other. Then, under the abovementioned conditions, the filtering problem of the stochastic dynamic system is to determine the optimal estimated value $\widehat{x}_{t}$ of the latent state $x_{t}$ based on a given observation sequence $Y_{t}=\left\{y_{1}, y_{2}, \ldots, y_{t}\right\}$. When solving the filtering problem, the most important thing is to determine the conditional probability density function $p\left(x_{t} \mid Y_{t}\right)$. Because once $p\left(x_{t} \mid Y_{t}\right)$ is known, it is easy to determine the optimal estimate $\hat{x}_{t}$ of $x_{t}$.

The method to solve the randomness of observation information can be obtained from [25].

3.2.2. Modeling Assumptions. This paper assumes that the working state of the rolling bearing is divided into a normal operating stage and defective operating stage. $l_{1}$ and $l_{2}$ are the duration of the two stages, respectively. The stages are assumed to be statistically independent.

Also assume the defect occurrence point $u$ can be identified, the rolling bearing has no maintenance intervention during operation, and during the defect operating stage, $p\left(y_{i} \mid r_{i}\right)$ describes the relationship between current and historical monitoring data $y_{i}$ and remaining life $r_{i}$.

3.2.3. Modeling Method. There is a negative correlation in the defect operating stages, while there is no definite relationship between the monitoring data and the life span in the normal operating condition. Therefore, the modeling for remaining life prediction is performed for the defective operating stage. A probability density function $f(y i, \alpha, \beta)$, $\alpha>0, \beta>0$, characterizes the monitoring data $y_{i}$ obeying the two-parameter distribution, where $\alpha$ and $\beta$ are scale and shape parameter, respectively. The relationship between current monitoring data $y_{i}$ and remaining life $r_{i}$ is given by

$$
p\left(y_{i} \mid r_{i}\right)= \begin{cases}p\left(y_{i}\right)=f\left(y_{i} ; \alpha, \beta\right), & \text { normal operating stage, } \\ p\left(y_{j}^{\prime} \mid r_{j}^{\prime}\right)=f\left(y_{j}^{\prime} ; \alpha^{\prime}, \beta^{\prime}\right), & \text { defective operating stage. }\end{cases}
$$

The distribution form of $p\left(y_{i} \mid r_{i}\right)$ can be chosen freely. Weibull distribution, Gamma distribution, and Lognormal distribution are all optional distribution forms. Research [33] showed that the difference between different distribution forms is not much. Weibull distribution is probably the most widely used form of failure time distribution. The closed form of the Weibull survivor function and the variety of shapes exhibited by the Weibull probability density function make it particularly convenient for generalizing exponential distribution [34]. Therefore, this paper uses the two-parameter Weibull distribution.

Secondly, the relationship $p\left(r_{j}^{\prime} \mid y_{j}^{\prime}\right)$ between the monitoring data $y_{j}^{\prime}$ and the remaining life $r_{i}$ is established from the defect start time to the current time. For the remaining life, the relationship is as follows:

$$
r_{i}= \begin{cases}r_{i-1}-\left(t_{i}-t_{i-1}\right), & r_{i-1}>t_{i}-t_{i-1} \\ 0, & r_{i-1} \leq t_{i}-t_{i-1}\end{cases}
$$

The stochastic filtering theory is used to establish the model $p\left(r_{j}^{\prime} \mid y_{j}^{\prime}\right)$, as shown below.
Since $y_{j}^{\prime}=\left\{y_{1}^{\prime}, \ldots, y_{j}^{\prime}\right\}, p\left(r_{j}^{\prime} \mid y_{j}^{\prime}\right)$ can be expressed as $p\left(r_{j}^{\prime} \mid y_{j}^{\prime}, y_{j-1}^{\prime}\right)$.

From the conditional probability formula, the model can be obtained as

$$
\begin{aligned}
p\left(r_{j}^{\prime} \mid y_{j}^{\prime}\right) & =p\left(r_{j}^{\prime} \mid y_{j}^{\prime}, y_{j-1}^{\prime}\right)=\frac{p\left(r_{j}^{\prime}, y_{j}^{\prime} \mid y_{j-1}^{\prime}\right)}{p\left(y_{j} \mid y_{j-1}^{\prime}\right)}, \\
p\left(r_{j}^{\prime}, y_{j}^{\prime} \mid y_{j-1}^{\prime}\right) & =p\left(y_{j}^{\prime} \mid r_{j}^{\prime}, y_{j-1}^{\prime}\right) p\left(y_{j} \mid y_{j-1}^{\prime}\right), \\
p\left(y_{j}^{\prime} \mid r_{j}^{\prime}, y_{j-1}^{\prime}\right) & =p\left(y_{j}^{\prime} \mid r_{j}^{\prime}\right) .
\end{aligned}
$$

that is, when $r_{j}^{\prime}$ and $y_{j-1}^{\prime}$ are given at the same time, $y_{i}$ only depends on $r_{j}^{\prime}$, so

$$
p\left(r_{j}^{\prime}, y_{j}^{\prime} \mid y_{j-1}^{\prime}\right)=p\left(y_{j}^{\prime} \mid r_{j}^{\prime}\right) p\left(r_{j}^{\prime} \mid y_{j-1}^{\prime}\right) \text {. }
$$

From the full probability formula, 


$$
\begin{aligned}
p\left(y_{j}^{\prime} \mid y_{j-1}^{\prime}\right) & =\int_{0}^{\infty} p\left(y_{j}^{\prime} \mid r_{j}^{\prime}, y_{j-1}^{\prime}\right) p\left(r_{j}^{\prime} \mid y_{j-1}^{\prime}\right) \mathrm{d} r_{j}, \\
& =\int_{0}^{\infty} p\left(y_{j}^{\prime} \mid r_{j}^{\prime}\right) p\left(r_{j}^{\prime} \mid y_{j-1}^{\prime}\right) \mathrm{d} r_{j}^{\prime} .
\end{aligned}
$$

According to the determination rules of the distribution of random variable functions, the following expression can be established:

$$
p\left(r_{j}^{\prime} \mid y_{j-1}^{\prime}\right)=p\left(r_{j-1} \mid r_{j-1}^{\prime}>t_{j}^{\prime}, y_{j-1}^{\prime}\right) \frac{\partial r_{j-1}^{\prime}}{\partial r_{j}^{\prime}} .
$$

As $r_{j-1}^{\prime}=r_{j}^{\prime}+t_{j}^{\prime}-t_{j-1}^{\prime}, \partial r_{j-1}^{\prime} / \partial r_{j}^{\prime}=1$. Then, the flowing equation can be obtained:

$$
p\left(r_{j-1} \mid r_{j-1}^{\prime}>t_{j}^{\prime}, y_{j-1}^{\prime}\right)=\frac{p\left(r_{j-1}^{\prime} \mid y_{j-1}^{\prime}\right)^{\infty}}{\int_{t_{j}^{\prime}}-t_{j-1}^{\prime}} p\left(r_{j-1}^{\prime} \mid y_{j-1}^{\prime}\right) \mathrm{d} r_{j-1}^{\prime},
$$

$$
\begin{aligned}
p\left(r_{j}^{\prime} \mid y_{j-1}^{\prime}\right) & =\frac{p\left(r_{j-1}^{\prime} \mid y_{j-1}^{\prime}\right)^{\infty}}{\int_{t_{j}^{\prime}}-t_{j-1}^{\prime}} p\left(r_{j-1}^{\prime} \mid y_{j-1}^{\prime}\right) \mathrm{d} r_{j-1}^{\prime} \\
= & \frac{p\left(r_{j-1}^{\prime}=r_{j}^{\prime}+t_{j}^{\prime}-t_{j-1}^{\prime} \mid y_{j-1}^{\prime}\right)^{\infty}}{\int_{t_{j}^{\prime}}-t_{j-1}^{\prime}} p\left(r_{j-1}^{\prime} \mid y_{j-1}^{\prime}\right) \mathrm{d} r_{j-1}^{\prime} .
\end{aligned}
$$

Therefore, if the distribution of initial conditions $p\left(r_{0}^{\prime}\right)$ and $p\left(y_{i} \mid r_{i}\right)=f\left(y_{i} ; \alpha_{1} ; \beta_{1}\right)$ are known, $p\left(r_{j}^{\prime} \mid y_{j}^{\prime}\right)$ can be obtained by recursive calculation of the stochastic filtering equation:

$$
\begin{aligned}
p\left(r_{j}^{\prime} \mid y_{j}^{\prime}\right) & =\frac{p\left(y_{j}^{\prime} \mid r_{j}^{\prime}\right) p\left(r_{j-1}^{\prime} \mid y_{j-1}^{\prime}\right)}{\int_{0}^{\infty} p\left(y_{j}^{\prime} \mid r_{j}^{\prime}\right) p\left(r_{j}^{\prime}+t_{j}^{\prime}-t_{j-1}^{\prime} \mid y_{j-1}^{\prime}\right) \mathrm{d} r_{j}^{\prime}}, \\
& =\frac{p\left(y_{j}^{\prime} \mid r_{j}^{\prime}\right) p\left(r_{j}^{\prime}+t_{j}^{\prime}-t_{j-1}^{\prime} \mid y_{j-1}^{\prime}\right)}{\int_{0}^{\infty} p\left(y_{j}^{\prime} \mid r_{j}^{\prime}\right) p\left(r_{j}^{\prime}+t_{j}^{\prime}-t_{j-1}^{\prime} \mid y_{j-1}^{\prime}\right) \mathrm{d} r_{j}^{\prime}} .
\end{aligned}
$$

For general conditions

$$
\begin{aligned}
p\left(r_{j}^{\prime} \mid y_{j}^{\prime}\right) & =\frac{p\left(y_{j}^{\prime} \mid r_{j}^{\prime}\right) p\left(r_{j-1}^{\prime} \mid y_{j-1}^{\prime}\right)}{\int_{0}^{\infty} p\left(y_{j}^{\prime} \mid r_{j}^{\prime}\right) p\left(r_{j-1}^{\prime} \mid y_{j-1}^{\prime}\right) \mathrm{d} r_{j}^{\prime}}, \\
& =\frac{\prod_{i=1}^{j} p\left(y_{j}^{\prime} \mid r_{i}^{\prime}\right) p\left(r_{j}^{\prime}+t_{j}^{\prime}-t_{0}^{\prime}\right)}{\int_{0}^{\infty} \prod_{i=1}^{j} p\left(y_{j}^{\prime} \mid r_{i}^{\prime}\right) p\left(r_{j}^{\prime}+t_{j}^{\prime}-t_{0}^{\prime}\right) \mathrm{d} r_{j}^{\prime}} .
\end{aligned}
$$

Equation (14) is the RUL prediction model. In the defective operating stage, the remaining life can be predicted from the beginning of deterioration to current monitoring data, thus supporting maintenance decisions.
3.2.4. Parameter Estimation. Here, the maximum likelihood estimation method is selected to estimate the relevant parameters. In practical applications, there are many state monitoring data and fewer failure data or life data. Since there are multiple parameters to be estimated, it is difficult to process. The model estimates the unknown parameters in the following three steps.

(1) Estimation of the unknown parameters in a given distribution $f_{1}\left(l_{1}\right)$ and $f_{2}\left(l_{2}\right)$ with the life data of normal operating stage and defective operating stage.

(2) Estimation of unknown parameters in $p\left(y_{i} \mid r_{i}\right)=$ $f\left(y_{i} ; \alpha_{1} ; \beta_{1}\right)$ that requires the historical monitoring data of normal operating stage.

(3) Estimation of unknown parameters in $p\left(y_{i}^{\prime} \mid r_{i}^{\prime}\right)=$ $f\left(y_{i}^{\prime} ; \alpha^{\prime}\left(r_{j}^{\prime}\right) ; \beta^{\prime}\right)$ that requires the life data of defective operating stage and the historical data of state monitoring for estimating by the maximum likelihood method.

3.3. Two Stage Multiparameter Fusion Stochastic Filtering Prediction Method. This paper proposes a two-stage stochastic filtering model for RUL prediction with multiparameter fusion. Multifeatures are extracted from the time domain, frequency domain, and complexity angle. Then, spectral regression is used to reduce the dimension and find the internal structure of the data. Based on the normal operating condition data, the baseline GMM is established. The distance BID based on Bayesian inference between the test data and the established GMM is calculated as the global monitoring indicator of bearing performance degradation. The control limit at the normal operating state is established by KDE. The absence of an overlimit means that the bearing is still running normally, and the monitoring and calculation of its global monitoring indicators work continuously. An overlimit indicates that the bearing enters the defective operating stage, and the stochastic filtering model predicts the remaining life dynamically. Figure 3 gives the flow diagram of the proposed prediction process.

\section{Experimental Results}

4.1. Prognosis Based on the Method Proposed in This Paper. A characteristic parameter alone cannot reflect the state change of the rolling bearing comprehensively, and for the same rolling bearing, different characteristic parameters have different change trends. However, the multiparameter fusion can overcome these problems because it can characterize the state of the bearing comprehensively and sensitively and detects the symptoms of the defect earlier. Therefore, to obtain the quantitative indicator BID as the input of the model, this paper uses multicharacteristic parameters for fusion.

In order to verify the effectiveness of the method proposed in this paper, the rolling bearing accelerated fatigue life experiment was carried out on an ABLT-1A tester. The test rig is shown in Figure 4. The experimental setup and structure of electrostatic sensors are shown in the literature [27]. In this experiment, six bearings were tested. The bearing failure modes in this experiment include rolling ball fault, inner race 


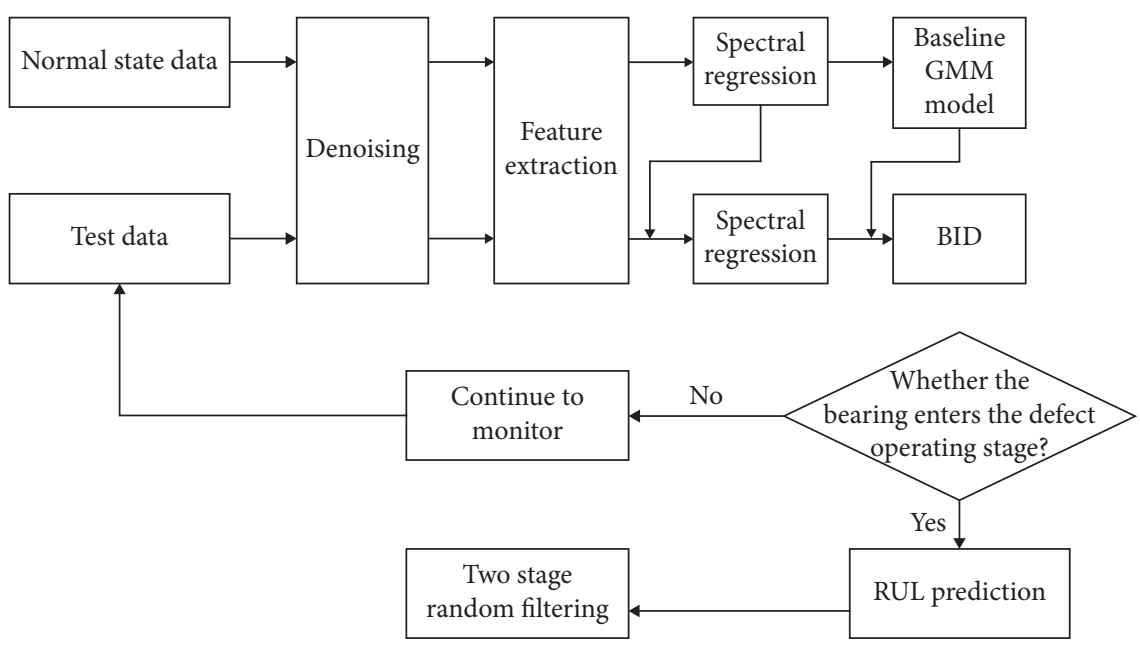

Figure 3: Remaining useful life prediction framework based on multiparameter fusion.

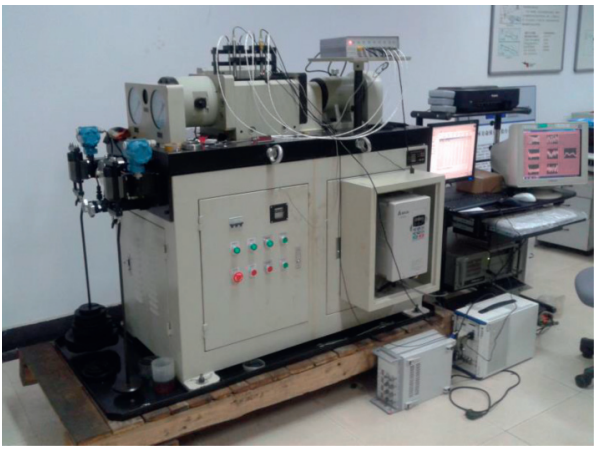

FIgURE 4: Test rig.

fault, and outer race fault. Among the 6 bearing experiments, four bearings had inner ring fault, one bearing had outer ring fault, and one bearing had rolling ball fault.

Taking bearing $\mathrm{B} 1$ and $\mathrm{B} 2$ as examples, RMS, kurtosis, and permutation entropy were extracted as feature parameters [27]. It can be seen that single feature parameter is not sensitive or consistent to the degradation of bearing performance in Figures 5(a)-5(c). SR-GMM-BID was adopted to fuse multiple features, and the results are shown in Figure 5(d). It can be seen that the BID index proposed in this paper can integrate multiple feature characteristics to find the occurrence of early bearing degradation earlier. The specific process can be obtained from our previous work [27].

Figure 6 shows the BID indicator for six rolling bearings. Although the operating environment and conditions are the same, the operational lifetime of the rolling bearing shows great randomness. Moreover, in the early stage of rolling bearing operation, the BID evaluation indicator is relatively stable. With the generation and development of defects, the BID value increases rapidly until the failure occurs, and the operation process has two stages. The lifetime of a bearing in normal operating condition ranges from 3,000 to 10,000 minutes. It also shows great randomness. Therefore, the first stage is mainly monitored, and there is no need for life prediction. Once the bearing enters the degeneration period (defective operating stage), the calculation of the remaining life starts.
Firstly, select the distribution form of $l_{1}, l_{2}$, and $y_{i}$. Using the Weibull distribution, the probability density functions are as follows:

$$
\begin{aligned}
f_{1}\left(l_{1}\right) & =\lambda_{1} \eta_{1}\left(\lambda_{1} l_{1}\right)^{\eta_{1}-1} e^{-\left(\lambda_{1} l_{1}\right)^{\eta_{1}},}, \\
f_{2}\left(l_{2}\right) & =\lambda_{2} \eta_{2}\left(\lambda_{2} l_{2}\right)^{\eta_{2}-1} e^{-\left(\lambda_{2} l_{2}\right)^{\eta_{2}},}, \\
p\left(y_{i}\right) & =\alpha \beta\left(\alpha y_{i}\right)^{\beta-1} e^{-\left(\alpha y_{i}\right)^{\beta}}, \\
p\left(y_{i}^{\prime} \mid r_{i}^{\prime}\right) & =\alpha^{\prime} \beta^{\prime}\left(\alpha^{\prime} y_{i}^{\prime}\right)^{\beta^{\prime}-1} e^{-\left(\alpha^{\prime} y_{i}^{\prime}\right)^{\beta^{\prime}} .} .
\end{aligned}
$$

Secondly, establish the relationship between the monitoring data $y_{i}^{\prime}$ of the defect operating stage and the remaining life $r_{i}$.

In normal operating stage, the measured data $y_{i}$ fluctuates within a certain range, there is no overall change, and the remaining life is decreasing. The measured data has no certain relationship with the remaining life, which can be expressed as

$$
E\left(y_{i} \mid x_{i}=2\right) \propto \frac{1}{\alpha_{2}}=\frac{1}{\alpha_{1}} e^{b\left(t_{i}-l_{1}\right)^{c}} .
$$

In the defect operating stage, the monitoring data has an overall upward trend, and the remaining life is decreasing. Assume that at the moment $t_{i}$, the negative exponential relationship describes the relationship of the increase of the monitoring data $y_{i}^{\prime}$ with the decrease in remaining life $r_{i}$. The expected value of monitoring data at normal operating condition should be approximately equal to the intercept of the expected value of monitoring data at the defect operating stage, which can be expressed as

$$
E\left(y_{i} \mid x_{i}=2\right) \propto \frac{1}{\alpha_{2}}=\frac{1}{\alpha_{1}} e^{b\left(t_{i}-l_{1}\right)^{c}},
$$

that is,

$$
\alpha_{2}=\frac{\alpha_{1}}{e^{b\left(t_{i}-l_{1}\right)^{c}}} .
$$

The maximum likelihood estimation method is used to estimate the unknown parameters in the model. Table 1 lists the estimated values of each parameter. The Bearing 1 lifetime test is taken as an example of the analysis. Figure 7 


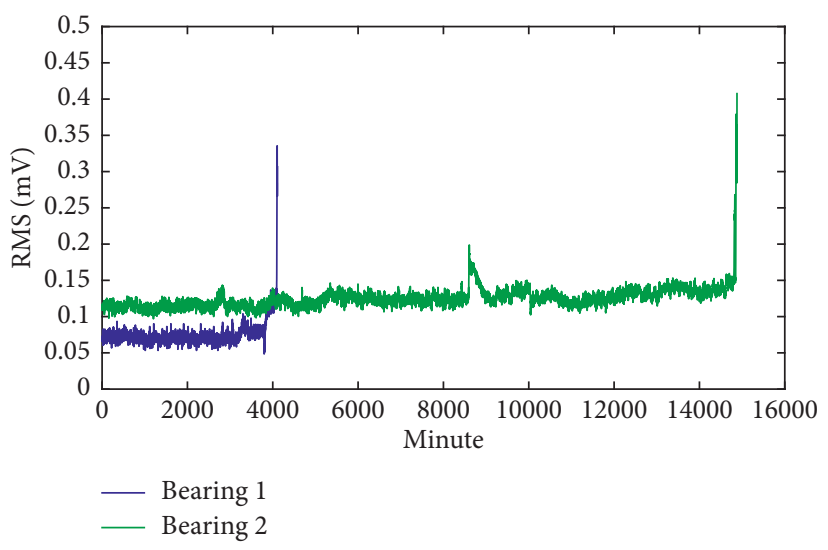

(a)

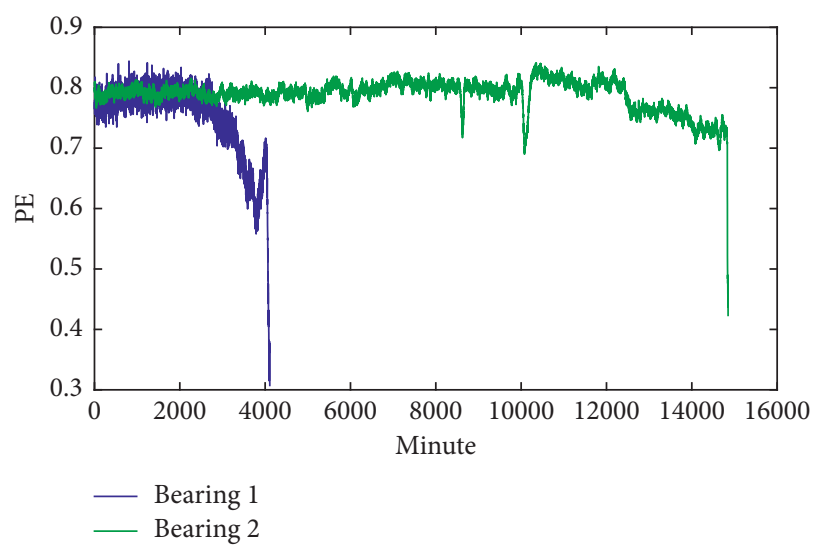

(c)

Figure 5: Trend charts of different indicators. (a) RMS. (b) Kurtosis. (c) Permutation entropy. (d) BID.

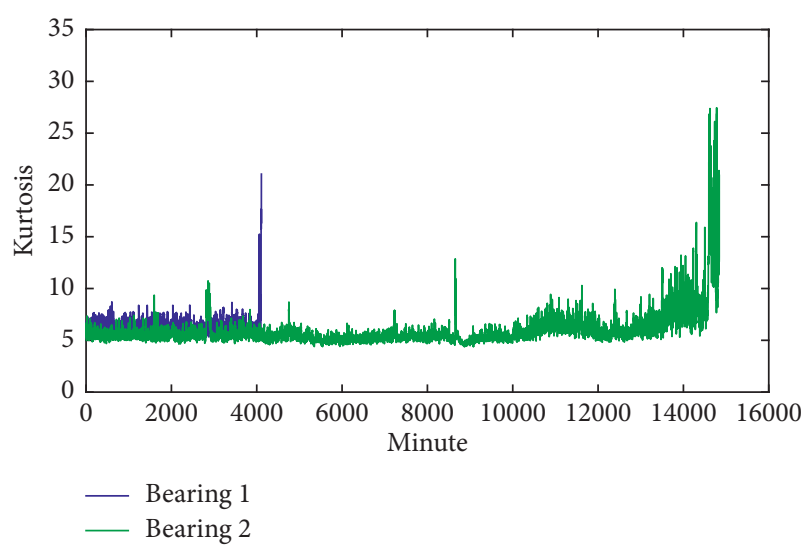

(b)

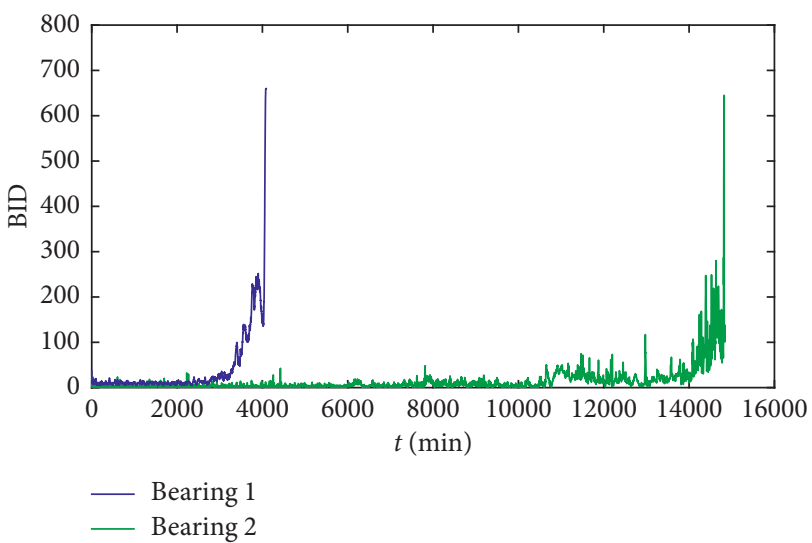

(d)

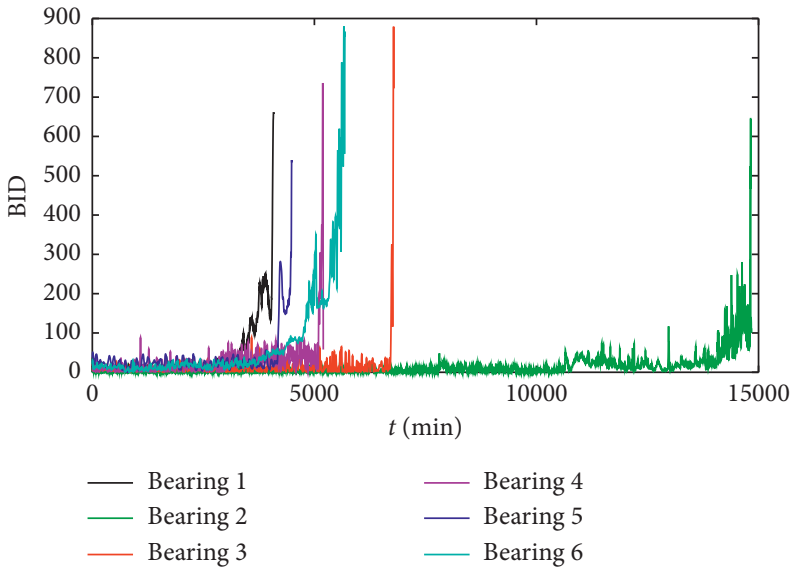

Figure 6: BID trend chart of six bearing life tests.

shows the results of RUL prediction based on multifeature parameter fusion in different moments of the defective operating stage. Red marks in the figure indicate the actual remaining life at that moment.

From Figure 7, a relatively small change of abnormal signal in the early stage of the defective operating stage leads to a large predicted error (the difference between actual remaining life and expected life expectancy value) of the remaining life, and there is a wide distribution range of RUL and higher uncertainty. However, as more signals are collected, the distribution of remaining life narrows, and the uncertainty and the prediction error decrease. In particular, at the last state monitoring point, the predicted remaining life of 180 minutes is very close to its actual remaining life of 200 minutes.

4.2. Comparison and Analysis. The prediction method based on hidden semi-Markov model (HSMM) also does not need to know the failure threshold [35]. This section compares the method proposed in this paper with the prediction method based on HSMM. The first three dimensional feature vector extracted by spectral regression is used as the data input to solve the life prediction problem of rolling bearing.

HSMM is trained with the life-cycle data of the second to sixth experiments. Taking the first experiment data as the test sample, the transition probabilities between three different states, as well as the mean and variance of the dwell time of each state are estimated by life-cycle experiment estimation (Tables 2 and 3).

Ten test samples are randomly selected from the samples of the first life-cycle experiment (due to the large deviation of the normal state, there is no significance in life prediction, so samples of initial degradation state and severe degradation state are selected). Table 4 lists the results of RUL prediction of the 10 test samples using HSMM. In the 10 test samples, 
TABLE 1: Estimated values of the stochastic filtering model parameters.

\begin{tabular}{|c|c|c|c|c|c|c|c|c|c|}
\hline Parameters & $\hat{\lambda_{1}}$ & $\hat{\eta_{1}}$ & $\hat{\lambda_{2}}$ & $\hat{\eta}_{2}$ & $\widehat{\alpha}$ & $\widehat{\beta}$ & $\hat{\beta^{\prime}}$ & $\widehat{B}$ & $\widehat{C}$ \\
\hline Estimated values & 4329.8 & 2.2849 & 716.9 & 0.7901 & 14.0403 & 1.1131 & 1.3339 & 197.1140 & 0.0657 \\
\hline
\end{tabular}

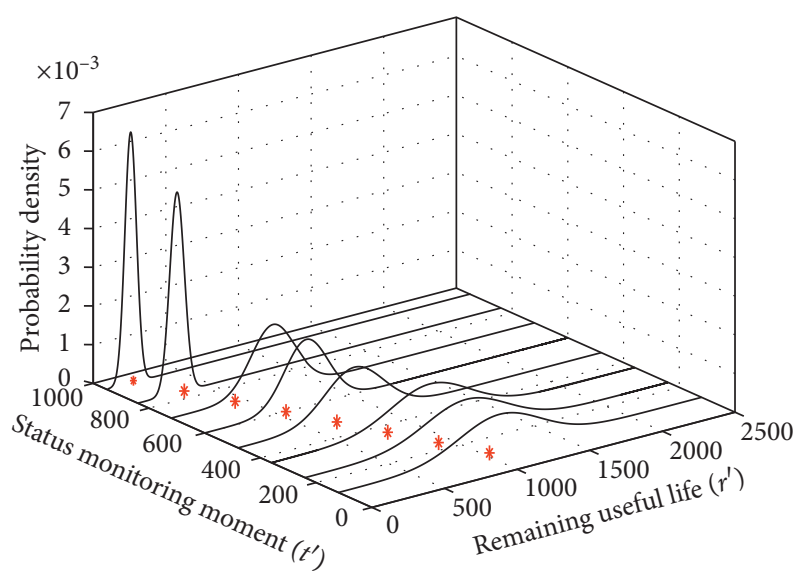

FIGURE 7: Probability density curve of RUL prediction for Bearing 1 at different times.

TABLE 2: State transition probabilities.

\begin{tabular}{lccc}
\hline & State 1: normal state & State 2: initial degradation state & State 3: severe degradation state \\
\hline State 1: normal state & 0.8853 & 0.1147 & 0 \\
State 2: initial degradation state & 0 & 0.7537 & 0.2463 \\
State 3: severe degradation state & 0 & 0 & 1 \\
\hline
\end{tabular}

TABle 3: Residence time for each state.

\begin{tabular}{lccc}
\hline Type of state & Normal state & Initial degradation state & Severe degradation state \\
\hline Mean & 4531 & 1048 & 438 \\
Variance & 1442 & 529 & 235 \\
\hline
\end{tabular}

TABLE 4: RUL prediction results based on HSMM.

\begin{tabular}{|c|c|c|c|c|c|c|c|}
\hline $\begin{array}{l}\text { Sampling } \\
\text { points }\end{array}$ & $\begin{array}{c}\text { Degradation } \\
\text { state }\end{array}$ & $\begin{array}{l}\text { Identification of } \\
\text { degradation state }\end{array}$ & $\begin{array}{c}\text { Mean of the } \\
\text { predicted result }\end{array}$ & $\begin{array}{l}\text { Variance of the } \\
\text { predicted result }\end{array}$ & $\begin{array}{l}\text { Interval of the } \\
\text { predicted result }\end{array}$ & $\begin{array}{c}\text { Actual } \\
\text { RUL }\end{array}$ & $\begin{array}{c}\text { Predicted } \\
\text { RUL }\end{array}$ \\
\hline 1 & $\begin{array}{c}\text { Initial } \\
\text { degradation }\end{array}$ & Normal state & 5238 & 1910 & {$[3328,7148]$} & 910 & Inaccurate \\
\hline 2 & $\begin{array}{c}\text { Initial } \\
\text { degradation }\end{array}$ & Initial degradation & 1227 & 633 & {$[594,1860]$} & 805 & Accurate \\
\hline 3 & $\begin{array}{c}\text { Initial } \\
\text { degradation }\end{array}$ & Initial degradation & 1227 & 633 & {$[594,1860]$} & 785 & Accurate \\
\hline 4 & $\begin{array}{c}\text { Initial } \\
\text { degradation }\end{array}$ & Initial degradation & 1227 & 633 & {$[594,1860]$} & 753 & Accurate \\
\hline 5 & $\begin{array}{c}\text { Initial } \\
\text { degradation }\end{array}$ & Severe degradation & 327 & 115 & {$[212,442]$} & 717 & Inaccurate \\
\hline 6 & $\begin{array}{c}\text { Initial } \\
\text { degradation }\end{array}$ & Initial degradation & 1227 & 633 & {$[594,1860]$} & 648 & Accurate \\
\hline 7 & $\begin{array}{c}\text { Initial } \\
\text { degradation }\end{array}$ & Initial degradation & 1227 & 633 & {$[594,1860]$} & 599 & Accurate \\
\hline 8 & $\begin{array}{c}\text { Severe } \\
\text { degradation }\end{array}$ & Severe degradation & 327 & 115 & {$[212,442]$} & 303 & Accurate \\
\hline 9 & $\begin{array}{c}\text { Severe } \\
\text { degradation }\end{array}$ & Severe degradation & 327 & 115 & {$[212,442]$} & 210 & Accurate \\
\hline 10 & $\begin{array}{c}\text { Severe } \\
\text { degradation }\end{array}$ & Severe degradation & 327 & 115 & {$[212,442]$} & 116 & Inaccurate \\
\hline
\end{tabular}


two initial degradation states are identified incorrectly, resulting in inaccurate life prediction, and one severe degradation state is inaccurate. The remaining 7 test samples have correct prediction results, which basically met the needs of remaining life prediction.

By comparing the prediction method of RUL based on HSMM and stochastic filtering, it can be seen that

(1) Both of the two methods need failure history data for model training and parameter estimation.

(2) HSMM needs to divide the life cycle into several different degradation stages, which has the characteristics of subjective factors and no definite rule to follow.

(3) HSMM supports the input of multidimensional characteristic parameters, while stochastic filtering generally supports the input of single-dimensional characteristic parameters. The input of multidimensional characteristic parameters needs calculation the joint probability distribution, which will increase the calculation amount and is not suitable for online learning. In this paper, the multidimensional characteristic parameters are effectively integrated into the quantitative indicator of reaction performance degradation and used as the input of the random filter model to solve this problem.

(4) It can be concluded from the prediction results that RUL prediction results may be greatly deviated due to the inaccurate state identification of HSMM. At the same time, because the next state of HSMM is only related to the current state and the hypothesis that has nothing to do with the history data does not conform to reality, the variance of the prediction results is large, and the uncertainty is high. The stochastic filtering model integrates the historical observation data and establishes the relationship between the observation value and RUL, so the accuracy of the prediction results is relatively high, especially in the stage of severe degradation.

\section{Conclusion}

In this paper, based on the electrostatic monitoring data, a modified stochastic filtering remaining useful life prediction method based on multifeature parameter fusion is proposed for the two-stage nonlinear degradation of rolling bearing operation. Monitoring is dominant at normal operating conditions, while the life prediction starts when the defect occurs. In the remaining life prediction, BID is constructed as a quantitative indicator for the degradation degree of bearing performance degradation degree. Then, taking it as input, the relationship between BID and RUL is established by the two-stage stochastic filtering model to realize online dynamic RUL prediction. This method solves the problem that the failure threshold of the rolling bearing is difficult to be accurately defined, and the problem that the joint probability distribution of the stochastic filtering model needs to be calculated when high-dimensional data is input. The experimental results show that the proposed method has simple calculation process and high prediction accuracy and engineering value.

\section{Data Availability}

All data can be obtained from the corresponding author upon request.

\section{Conflicts of Interest}

The authors declare that they have no conflicts of interest.

\section{Acknowledgments}

This research was supported by the National Natural Science Foundation of China, grant number 51505229 and Fundamental Research Funds for the Central Universities, NJ2019014.

\section{References}

[1] T. J. Harvey, R. J. K. Wood, and H. E. G. Powrie, "Electrostatic wear monitoring of rolling element bearings," Wear, vol. 263, no. 7-12, pp. 1492-1501, 2007.

[2] M. Craig, T. J. Harvey, R. J. K. Wood et al., "Advanced condition monitoring of tapered roller bearings, part 1," Tribology International, vol. 42, no. 11-12, pp. 1846-1856, 2009.

[3] S. L. Chen, R. J. K. Wood, L. Wang, R. Callan, and H. E. G. Powrie, "Wear detection of rolling element bearings using multiple-sensing technologies and mixture-modelbased clustering method," Proceedings of the Institution of Mechanical Engineers, Part O: Journal of Risk and Reliability, vol. 222, no. 2, pp. 207-218, 2008.

[4] O. D. Tasbaz, R. J. K. Wood, M. Browne, H. E. G. Powrie, and G. Denuault, "Electrostatic monitoring of oil lubricated sliding point contacts for early detection of scuffing," Wear, vol. 230, no. 1, pp. 86-97, 1999.

[5] S. Morris, R. J. K. Wood, T. J. Harvey et al., "Electrostatic charge monitoring of unlubricated sliding wear of a bearing steel," Wear, vol. 255, no. 1-6, pp. 430-443, 2003.

[6] L. Wang, R. J. K. Wood, T. J. Harvey et al., "Wear performance of oil lubricated silicon nitride sliding against various bearing steels," Wear, vol. 255, no. 1-6, pp. 657-668, 2003.

[7] L. Wang, I. Wood, and H. E. G. Powrie, "Electrostatic wear sensing of ceramic-steel lubricated contacts," Tribology Series, vol. 43, pp. 711-720, 2003.

[8] X.-S. Si, W. Wang, C.-H. Hu, and D.-H. Zhou, "Remaining useful life estimation-a review on the statistical data driven approaches," European Journal of Operational Research, vol. 213, no. 1, pp. 1-14, 2011.

[9] Y. Qian, R. X. R. Yan, and R. X. Gao, "A multi-time scale approach to remaining useful life prediction in rolling bearing," Mechanical Systems and Signal Processing, vol. 83, pp. 549-567, 2017.

[10] G. J. Kacprzynski, A. Sarlashkar, M. J. Roemer, A. Hess, and B. Hardman, "Predicting remaining life by fusing the physics of failure modeling with diagnostics," JOM, vol. 56, no. 3, pp. 29-35, 2004.

[11] Y. Liu and S. Mahadevan, "Probabilistic fatigue life prediction using an equivalent initial flaw size distribution," International Journal of Fatigue, vol. 31, no. 3, pp. 476-487, 2009. 
[12] C. N. Giap, L. H. Son, and F. Chiclana, "Dynamic structural neural network," Journal of Intelligent \& Fuzzy Systems, vol. 34, no. 4, pp. 2479-2490, 2018.

[13] J. J. Rubio, "SOFMLS: online self-organizing fuzzy modified least-squares network," IEEE Transactions on Fuzzy Systems, vol. 17, no. 6, pp. 1296-1309, 2009.

[14] J. J. Rubio, D. Ricardo Cruz, I. Elias et al., "ANFIS system for classification of brain signals," Journal of Intelligent \& Fuzzy Systems (Preprint), vol. 37, no. 3, pp. 4033-4041, 2019.

[15] B. Yang, E. R. Liu, and E. Zio, "Remaining useful life prediction based on a double-convolutional neural network architecture," IEEE Transactions on Industrial Electronics, vol. 66, no. 12, pp. 9521-9530, 2019.

[16] J. Carroll, S. Koukoura, A. McDonald, A. Charalambous, S. Weiss, and S. McArthur, "Wind turbine gearbox failure and remaining useful life prediction using machine learning techniques," Wind Energy, vol. 22, no. 3, pp. 360-375, 2019.

[17] W. Li, Z. Jiao, L. Du, W. Fan, and Y. Zhu, "An indirect RUL prognosis for lithium-ion battery under vibration stress using Elman neural network," International Journal of Hydrogen Energy, vol. 44, no. 23, pp. 12270-12276, 2019.

[18] C. Ordóñez, F. Sánchez Lasheras, J. Roca-Pardiñas, and F. J. d. C. Juez, "A hybrid ARIMA-SVM model for the study of the remaining useful life of aircraft engines," Journal of Computational and Applied Mathematics, vol. 346, pp. 184191, 2019.

[19] Q. Wang, B. Zhao, J. Chang, H. Ma, and G. Mao, "A method for rapidly evaluating reliability and predicting remaining useful life using two-dimensional convolutional neural network with signal conversion," Journal of Mechanical Science and Technology, vol. 33, no. 6, pp. 2561-2571, 2019.

[20] S. Mishra and O. A. Vanli, "Remaining useful life estimation with lamb-wave sensors based on wiener process and principal components regression," Journal of Nondestructive Evaluation, vol. 35, no. 1, p. 11, 2016.

[21] Q. Aisong, Z. Qinghua, H. Qin et al., "Remaining useful life prediction for rotating machinery based on optimal degradation indicator," Shock and Vibration, vol. 2017, Article ID 6754968, 12 pages, 2017.

[22] D. Wang, F. Yang, K.-L. Tsui, Q. Zhou, and S. J. Bae, "Remaining useful life prediction of lithium-ion batteries based on spherical cubature particle filter," IEEE Transactions on Instrumentation and Measurement, vol. 65, no. 6, pp. 1282-1291, 2016.

[23] Y. Lei, N. Li, S. Gontarz, J. Lin, S. Radkowski, and J. Dybala, “A model-based method for remaining useful life prediction of machinery," IEEE Transactions on Reliability, vol. 65, no. 3 , pp. 1314-1326, 2016.

[24] V. M. Catterson, J. Melone, and M. S. Garcia, "Prognostics of transformer paper insulation using statistical particle filtering of on-line data," IEEE Electrical Insulation Magazine, vol. 32, no. 1, pp. 28-33, 2016.

[25] W. Wang, "A two-stage prognosis model in condition based maintenance," European Journal of Operational Research, vol. 182, no. 3, pp. 1177-1187, 2007.

[26] J. J. Rubio, E. Garcia, G. Ochoa et al., "Unscented Kalman filter for learning of a solar dryer and a greenhouse," Journal of Intelligent \& Fuzzy Systems, vol. 37, no. 5, pp. 6731-6741, 2019.

[27] Y. Zhang, A. Wang, and H. Zuo, "Roller bearing performance degradation assessment based on fusion of multiple features of electrostatic sensors," Sensors, vol. 19, no. 4, 2019.

[28] Y. Wang, Y. Peng, Y. Zi et al., "An integrated Bayesian approach to prognositics of the remaining useful life and its application on bearing degradation problem," in IEEE
International Conference on Industrial Informatics, IEEE, Cambridge, UK, July 2015.

[29] D. Wang and K. L. Tsui, "Statistical modeling of bearing degradation signals," IEEE Transactions on Reliability, vol. 66, no. 4, pp. 1331-1344, 2018.

[30] Y. Lei, N. Li, L. Guo et al., "Machinery health prognostics: a systematic review from data acquisition to RUL prediction," Mechanical Systems and Signal Processing, vol. 104, pp. 799834, 2018.

[31] N. Li, Y. Lei, J. Lin et al., "An improved exponential model for predicting remaining useful life of rolling element bearings," IEEE Transactions on Industrial Electronics, vol. 62, no. 12, pp. 7762-7773, 2015.

[32] D. Wang, K. L. Tsui, and Q. Miao, "Prognostics and health management: a review of vibration based bearing and gear health indicators," IEEE Access, vol. 6, pp. 665-676, 2017.

[33] W. Wang, "A model to predict the residual life of rolling element bearings given monitored condition information to date," IMA Journal of Management Mathematics, vol. 13, no. 1, pp. 3-16, 2002.

[34] J. Galambos, The Asymptotic Theory of Extreme Order Statistics, Wiley, New York, NY, USA, 1978.

[35] M. Dong, D. He, P. Banerjee et al., "Equipment health diagnosis and prognosis using hidden semi-Markov models," The International Journal of Advanced Manufacturing Technology, vol. 30, no. 7-8, pp. 738-749, 2006. 